VS16-P02

\section{Tunable crystal structure and proton conductivity of lanthanide nitrilotris- methylphosphonates}

Inés Ruiz Salcedo ${ }^{1}$, Aurelio Cabeza Díaz ${ }^{1}$, Montse Bazaga García ${ }^{1}$, Rosario Mercedes Pérez Colodrero², Gary Hix ${ }^{2}$, Enrique Ramírez Losilla $^{1}$, Pascual Olivera Pastor ${ }^{1}$

1. Departamento Química Inorgánica, Cristalografía y Mineralogía. Facultad de Ciencias. Universidad de Málaga., Málaga, Spain

2. Wolverhampton School of Sciences. Faculty of Science and Engineering. University of Wolverhampton., Wolverhampton, United Kingdom

email: inesrs@uma.es

Metal phosphonates are multifunctional solids with remarkable stability and proton conducting properties owing to their structure is usually composed of extended hydrogen-bond networks that favor proton transfer pathways [1]. Moreover, these properties can be enhanced by appropriate modification of the synthesis conditions $[2,3]$.

In this communication, a new family of isostructural 2D layered compounds based on lanthanide nitrilotris-methylphosphonates is reported. These compounds have been isolated at room temperature and have the general formula $\mathrm{Ln}\left[\mathrm{N}\left(\mathrm{CH}_{2}\right)_{3}\left(\mathrm{PO}_{3} \mathrm{H}_{2}\right)_{2}\left(\mathrm{PO}_{3} \mathrm{H}\right)\left(\mathrm{H}_{2} \mathrm{O}\right)\right] \mathrm{SO}_{4} \cdot 2 \mathrm{H}_{2} \mathrm{O}(\mathrm{Ln}=\mathrm{Pr}, \mathrm{Nd}$, $\mathrm{Sm}, \mathrm{Eu}, \mathrm{Gd}, \mathrm{Tb}, \mathrm{Dy}, \mathrm{Ho}, \mathrm{Er}$ and $\mathrm{Yb})$. The coordination environment of $\mathrm{Ln}^{3+}$ is composed by eight oxygen atoms from three different ligands and two oxygens from bound waters. This connectivity creates positive charged layers connected to sulfate ions through hydrogen-bonds. These compounds show promising proton conductivity with values ranging between $7.6 \cdot 10^{-2}$ and $3.8 \cdot 10^{-2} \mathrm{~S} \cdot \mathrm{cm}^{-1}$ at $80{ }^{\circ} \mathrm{C}$ and $95 \% \mathrm{RH}$ and low activation energy corresponding to Grotthuss-type proton transfer mechanism. In addition, a structural transformation occurs at $\mathrm{T}>70{ }^{\circ} \mathrm{C}$ accompanied by a remarkable enhanced conductivity. Studies on the structure-properties relationships will be discussed.

References:

[1] Metal phosphonate Chemistry: from synthesis to applications. 1 st ed., edited by A. Clearfield, K. D. Demadis, RSC-Publishing, Cambridge.

[2] M. Bazaga-García, G. K. Angeli, K. E. Papathanasiou, I. R. Salcedo, P. Olivera-Pastor, E. R. Losilla, D. Choquesillo-Lazarte, Gary B. Hix, A. Cabeza, K. Demadis. (2016). Inorganic Chemistry, 55, 7414-7424.

[3] A. Cabeza, P. Olivera-Pastor, R. M. P. Colodrero. (2015).

Tailored Organic-Inorganic Materials. Eds. E. Brunet, J. L. Colón, A. Clearfield. Ed. John Wiley \& Sons, Inc. Ch. 4, 137-191.

Keywords: metal phosphonates, proton conductivity, crystal structure

\section{MS16-P03}

\section{Perovskite-type hybrid materials with lead halide complexes and piperidine methyl derivatives: Design, synthesis and structural analysis}

Pawel Socha ${ }^{1}$, Lukasz Dobrzycki ${ }^{1}$, Michal K. Cyranski ${ }^{1}$

1. Department of Chemistry, University of Warsaw, Warsaw, Poland email:psocha@chem.uw.edu.pl

Perovskite-type halide hybrid salts are intriguing materials. Depending on the size and shape of an organic cation it is possible to obtain $0-\mathrm{D}, 1-\mathrm{D}, 2-\mathrm{D}$ or

3-D architecture of polymerized inorganic complexes in the crystal structure. Physicochemical properties of these materials strictly depend on this architecture, kind of metal cation and halide anion, what gives many possibilities of modification depending on creators preferences. Therefore, hybrid salts are known as semiconductors, magnetics or solar cell components. [1] [2]

The aim of this project is design, synthesis and structural analysis of new organometallic systems of lead halides piperidine (and its derivatives). Based on the received results, it is available to propose the rules governing the crystal architecture of the hybrid salts.

Piperidine is a six-membered heterocyclic aliphatic amine, which may be relatively easily modified by substitution e.g. by methyl group in various position(s) in respect to the nitrogen atom. Size of the organic cation should affect crystal structure, because it can fill more space in the unit cell. Additionally, covering of amine group should have influence on structure, because of reduce accessibility atoms to create hydrogen bonds between organic and inorganic part.

Lead halide was chosen as an inorganic part of hybrid material. It is well known that lead halide creates octahedral complex of $\mathrm{PbX}_{6}^{-4}$, hence it was expected to receive various motifs of polymerized inorganic complexes like 1-D chains, 2-D layers or 3-D motifs. [1][2][3] Chlorides and bromides was used independently as halides.

During the studies, 9 crystals were obtained. All structures were determined by single crystal $x$-ray diffraction and additionally characterized by Raman spectroscopy. For systems containing lead bromide, 5 of them exhibit 1-D inorganic chain structure. Structural analysis showed that, few of them are isostructural despite the different position of the methyl group. Moreover, one of them form uncommon 3-D inorganic structure (Fig. 1), what is exception in the examinated series of systems with piperidine derivatives and lead bromide. In contrast to the previous systems, 3 obtained crystals with lead chloride represent only 2-D architecture, which draws attention to the influence of the size of used halogen anion.

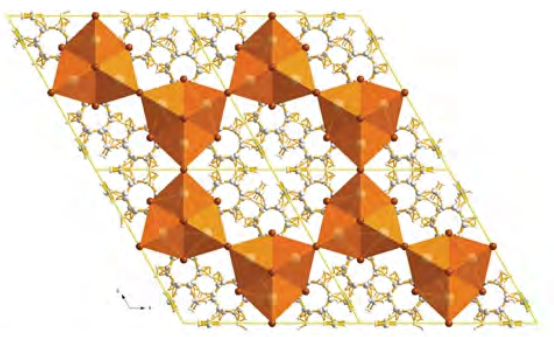


References:

[1] Liu, M., Johnston, M. B. \& Snaith, H. J. (2013). Nature, 501, 395-398

[2] Ye, H.-Y., Liao, W.-Q., Hu, C.-L., Zhang, Y., You, Y.-M., Mao, J.-G., Li, P.-F. \& Xiong, R.-G. (2016). Adv. Mater., 28, 2579-2586

[3] Dobrzycki, L. \& Wozniak, K. (2009). Journal of Molecular Structure 921, 18-33

Keywords: Hybrid materials, Perovskite-type halides, Crystal engineering

\section{MS16-P04}

\section{Pre-transitional processes in lead zirconate doped by Ti studied by diffuse and inelastic $\mathrm{X}$-ray scattering}

Daria Andronikova ${ }^{1}$, Roman Burkovsky ${ }^{2}$, Yurii Bronwald ${ }^{2}$, Alexei Bosak $^{3}$, Dmitry Chernyshov ${ }^{4}$, Igor Leontiev ${ }^{5}$, Nikolai Leontiev 6 , Alexey Filimonov ${ }^{2}$, Sergey Vakhrushe ${ }^{1}$

1. Ioffe Institute, St. Petersburg, Russia

2. Peter the Great St.Petersburg Polytechnic University, St. Petersburg, Russia

3. European Synchrotron Radiation Facility, Grenoble, France

4. Swiss-Norwegian Beamlines at the European Synchrotron Radiation Facility, Grenoble, France

5. Southern Federal University, Rostov-on-Don, Russia

6. Azov Black Sea Engineering Institute, Zernograd, Russia

email: andronikova.daria@gmail.com

Lead-zirconate titanate $\left(\mathrm{PbZr}_{1-\mathrm{x}} \mathrm{Ti}_{\mathrm{x}} \mathrm{O}_{3}, \mathrm{PZT}\right)$ is one of the most actively studied and widely used ferroelectric materials. One of the reasons of the interest is a complex phase diagram of lead zirconate and lead titanate solid solution, which illustrates variety of physical properties and crystal structures depending on titanium concentration. Other reasons of popularity are high piezolelectric properties, demonstrated by PZT around morthotropic phase boundary, and prospects of application of antiferrolectric properties [1], demonstrated by PZT with low Ti concentration.

Pure lead zirconate $(\mathrm{x}=0)$ is the prototypical antiferroelectric material. Between the cubic perovskite paraelectric phase and the antiferroelectric phase, in the narrow temperature range, intermediate ferroelectric phase exists. Addition of titanium increases the temperature range of stability of this phase. Cubic-to-intermediate phase transition is accompanied by doubling of the cell parameters of the paraelectric cubic lattice along two directions [2] and results in the appearance of M-superstructure with coordinate $(\mathrm{H} \pm 1 / 2$ $\mathrm{K} \pm 1 / 20$ ) in the diffraction pattern. Observation of additional satellites around M-point by electron diffraction [2] results in conclusion about complex domain pattern, characterized by antiphase domain boundary in lead displacement.

Recent studies [3] of pure lead zirconate reveals complex pattern of dynamical correlations in paraelectric phase. Diffuse scattering distribution indicates disordering of oxygen octahedral tilts and $\mathrm{Pb}$ displacements is shown in the high-temperature cubic phase. To study temperature behavior of these correlations X-ray diffuse scattering measurements have been done in wide temperature range in PZT with small titanium concentration $(\mathrm{x}<0.04)$. To characterize dynamical origin of DS lattice dynamics have been studied using inelastic X-ray scattering. Obtained temperature evolution of DS and pre-transitional dynamical peculiarities will be shown in presentation and discussed in the context of mode coupling.

Andronikova D. acknowledges support by Russian President Grants No. SP-3762.2018.5 